\title{
Adoption Gaps of Environmental Adaptation Technologies with Public Effects
}

\author{
Angelo Antoci ${ }^{1}$ (D) Simone Borghesi $i^{2,3} \cdot$ Giulio Galdi $^{3} \cdot$ Sergio Vergalli ${ }^{4,5}$
}

Accepted: 12 April 2021 / Published online: 2 June 2021

(c) The Author(s) 2021

\begin{abstract}
As the Intergovernmental Panel on Climate Change (2018) testifies, the world is a long way from halting climate change, let alone reverting it. The existence of adaptation and mitigation technologies did not prove sufficient, their adoption being respectively faulted or hindered by the presence of externalities. In this work, we study how externalities, whether positive or negative, lead the system away from Pareto-dominant (social optimum) states, towards Pareto-dominated ones. We show that adoption gaps, i.e. differences between socially optimum vs current adoption shares, of both (mal)adaptation and mitigation technologies are caused by the externalities emitted. In particular, over-adoption may occur for maladaptive technologies, whereas under-adoption may occur in case of mitigation. We employ a model with two regions at different stages of development and also derive relevant considerations on possible counterproductive effects of green policies and environmental dumping.
\end{abstract}

Keywords Maladaptation $\cdot$ Mitigation $\cdot$ Externalities $\cdot$ Environmental dumping

JEL Classification $\mathrm{C} 70 \cdot \mathrm{D} 62 \cdot \mathrm{O} 13 \cdot \mathrm{O} 40 \cdot \mathrm{Q} 20$

The authors would like to express their sincere appreciation to two anonymous reviewers for their very useful suggestions.

Angelo Antoci

antoci@uniss.it

1 Department of Economic and Business Sciences, University of Sassari, Sassari, Italy

2 Department of Political and International Sciences, University of Siena, Siena, Italy

3 Florence School of Regulation, European University Institute, Florence, Italy

4 Department of Economics and Management, University of Brescia, Brescia, Italy

5 Fondazione Eni Enrico Mattei (FEEM), Milan, Italy 


\section{Introduction}

Climate change and environmental hazards are exerting pressure on our societies at an increasing pace, requiring rapid and swift change in our economic systems in order to have a chance to stay below a $2^{\circ} \mathrm{C}$ degrees global temperature increase (Rogelj et al. 2018). We had to forgo the comforting idea that "natural systems fluctuate within an unchanging envelope of variability"(Milly et al. 2008). The scale of the challenge requires immediate action to cope with our rapidly changing climate. The IPCC and the UNEP ${ }^{1}$ characterise the possible responses to environmental and climatic hazards dichotomously, as either adaptation or mitigation strategies (UNEP 2019; IPCC 2014), with the implementation of one not excluding the other's. On the one hand, there are mitigation strategies at our disposal, which tackle the problem at its source and combat the causes of increased environmental risks. Efficient water management, restoration of soil, substitution of fossil fuels with agricultural by-products are all examples of mitigation techniques for the agricultural sector (Smith et al. 2007). Mitigation strategies not only reduce the environmental hazards for the adopter, but for all agents, thus generating a positive externality to other agents. On the other hand, adverse effects of climate change and environmental degradation can already be felt, prompting the development of solutions to deviate or alleviate the damages, i.e. adaptation strategies. Such strategies allow to cope with a changing climate, reducing the exposure of adopters to the ensuing harm. This includes responding to abnormally hot or cold temperatures, adopting new agricultural techniques to cope with the impoverishment of soil, creating artificial snow in ski resorts, and much more (for a broad review on many other forms of adaptation, see Tompkins et al. 2010). With respect to mitigation strategies, adaptation does not aim to reduce the problem, but rather to avoid at least part of its adverse affects. At times, this is done at the expense of other agents, i.e. adaptation strategies may generate negative externalities. An adaptation technology that exemplifies this dynamics and is recently gaining salience is solar geoengineering, whose aim is to reduce solar radiation on Earth. There are many techniques with which this could be attained, but all of them seem to carry their own flaw which could endanger vulnerable areas or communities (Reynolds 2019; Wagner and Weitzman 2015; Zhang et al. 2015). Indeed, the IPCC (2018) remarks that, according to extant knowledge on solar engineering, there are concerns of potential conflicts between the various techniques and sustainable development goals. Another notorious instance of maladaptation in the scientific literature are cooling and heating systems: by improving domestic temperature for the user, it increases the risk of energy shortages and ultimately worsens the problem of climate change (Lundgren and Kjellstrom 2013). These two notable examples and, in general, all strategies that shift environmental hazards onto others, postpone them for future generations, or disproportionally affect the most vulnerable, are defined by the literature as maladaptation, rather than simply as adaptation (Antoci et al. 2020; UNEP 2019; Barnett and O'Neill 2010). It should be noted that the UNEP (2019) stresses that every adaptation strategy that increases the opportunity cost of moving to a more sustainable alternative is maladaptation, as it has detrimental effects on long term sustainability. In this work we study the dynamics of mitigation and maladaptation, showing how global externalities lead to under-adoption of strategies of the former type and over-adoption of the latter.

${ }^{1}$ United Nations Environment Programme. 
Since mitigation strategies actually reduce environmental hazards instead of (temporarily) avoiding its effects, it is usually considered to be the most desirable strategy (IPCC 2014). However, there are reasons why humans did not respond with enthusiasm to the emergence of mitigation solutions in the face of environmental hazards. Firstly, many mitigation strategies require long-term investments to pay off, with a time scale that may exceed the average life expectancy of a person before they become effective (Hallegatte 2009). The incapacity of humans to make long-term investments and their preference for the present are additional threats to our capacity to make long-term commitments to stop environmental degradation (Warburton 2018), leading to issues of intergenerational equity (Glotzbach and Baumgartner 2012), which is a characterising feature of maladaptation (UNEP 2019). We remark that the existence of mitigation solutions is not a sufficient condition for the abatement of environmental damage. The literature has uncovered several ways in which externalities of any type, either negative or positive (as is the case for mitigation strategies) may undermine the achievement of the social optimum. On the one hand, whenever an agent may transfer her cost to protect against environmental hazards onto others, i.e. she emits negative externalities, in a way that has no consequences on herself, she has little incentive to adopt an existing mitigation strategy. For instance, an agent might prefer to install a substantially cheaper air conditioning system instead of investing to enhance house insulation. On the other hand, if a strategy actually reduces environmental risks not only for the adopter, but also for other agents, i.e. it has a positive externality, then it may happen that all agents wait for the others to tackle environmental degradation for everyone, but none is willing to pay the cost for the benefit of others ${ }^{2}$. This is but an instance of the well known free rider problem, which emerges from the non-excludability of agents from the benefits of a public good (Heller and Starrett 1976). Scholars studying these shiftable externalities highlighted that policy tools hindering maladaptive strategies and promoting mitigation ones are desirable, e.g. a tax on negative externalities or a subsidy on positive ones (Bird 1987; Shaw and Shaw 1991; Shogren and Crocker 1991; Geaun 1993).

In this work, we focus on the dichotomy between maladaptation and mitigation, studying the adoption dynamics of the related strategies. We assume that individuals from two regions at different stages of development have the possibility to adopt a technology which provides benefits for the adopters, but also generates externalities on other agents. In particular, each region has a local environmental indicator which is affected by the adoption dynamics of both regions, so that the externalities have a global effect. These externalities may be either negative, in case of a maladaptation technology, or positive, in case of a mitigation technology. We highlight what is the underlying mechanism that leads to over-adoption of maladaptation strategies and under-adoption of mitigation ones. In addition, we show what are the effects on the less developed region if the maladaptation technology is such that it disproportionally burdens its population with respect to the agents from the more developed region. Finally, we briefly analyse the case of a green policy in the developed region (abating local externalities) which has negative impacts on the environment in the less developed region (environmental dumping). We show that in this context the green policy may actually reduce the well-being of agents in both regions. The adoption dynamics of the technologies is modelled by a two

\footnotetext{
${ }^{2}$ In an experimental setting, Hasson et al. (2010) show that agents rarely contribute to the mitigation solution and that their contributions to a common mitigation policy are not sensitive to the likelihood of extremely adverse events. In a somewhat similar experiment, Milinski et al. (2006) show that reputation effects may nudge agents to contribute to a public good in an environmental framing.
} 
population evolutionary game which employs replicator equations, so that all agents may imitate their peers in the region, if the well-being of the latter is greater. Our analysis leads to three major conclusions: (1) when only a maladaptive technology is available, either all agents adopt it or none, depending on the initial distribution of strategies; (2) when only a mitigation technology is available, the system typically reaches a state in which a part of the population adopts the technology while the rest does not; no path dependency arises (3) if the more developed region dumps negative externalities onto the less developed one, it might happen the the well-being of all agents decreases. In Sect. 2 we illustrate our model. We then employ it to analyse the adoption dynamics of a maladaptive technology (Sect. 3) and of a mitigation one (Sect. 4). Finally, in Sect. 5 we elaborate on the results, draw some policy implications and sketch future research directions.

\section{The Model}

Let us consider two regions $j=N, S$. At any given time $t$, the well-being of agents from both regions depends on the quality of an environmental indicator, $E_{t}^{j}$, that is subject to be degraded or enhanced by other agents, i.e. by their externalities. Agents may decide to adopt an adaptation/mitigation strategy $\boldsymbol{A}$ to improve the personal benefit they derive from $E_{t}^{j}$, while being aware that they are in turn affected by the public effect, i.e. the externality, from the adaptation/mitigation strategies of other agents from the same region. One might think of neighbouring farmlands, where the land of one farmer can be threatened by the actions of others (e.g. volatile pesticides crossing over) or, vice versa, enhanced by them (e.g. neighbour's beehives increasing own crop fertility). Using pesticides or keeping a beehive are both actions that provide a private benefit to the adopter, while having an effect on the neighbouring farmland. In our model, this private effect is denoted by $p^{j}$ and is always positive. If agents decide not to use the adaptation/maladaptation technology, i.e. they choose strategy $N A$, they cannot enjoy the private effect. Independently from their chosen strategy, agents are also subject to public effect $P_{t}^{j}$, which is the summation of the externalities generated by agents adopting strategy $\boldsymbol{A}$ and is defined later in (2). Finally, the environmental quality of region $j$ when all agents in both countries adopt strategy $N A$ is equal to $\bar{E}^{j}$. The overall environmental quality for agents in $j=N, S$ adopting strategy $i=\boldsymbol{A}, \boldsymbol{N A}$ is thus described as follows:

$$
E_{t}^{j}= \begin{cases}\bar{E}^{j}+P_{t}^{j} & \text { if } i=\boldsymbol{N A} \\ \bar{E}^{j}+P_{t}^{j}+p^{j} & \text { if } i=\boldsymbol{A}\end{cases}
$$

where $P^{j}$ can take either sign. We define the well-being of an agent from region $j$ as the natural $\operatorname{logarithm}$ of $E^{j}$ minus the adoption $\operatorname{cost} C^{j}$ for agents choosing strategy $\boldsymbol{A}$ :

$$
\Pi_{i}^{j}:= \begin{cases}\ln \left(\bar{E}^{j}+P_{t}^{j}\right) & \text { if } i=\boldsymbol{N A} \\ \ln \left(\bar{E}^{j}+P_{t}^{j}+p^{j}\right)-C^{j} & \text { if } i=\boldsymbol{A}\end{cases}
$$

where the adoption cost $C^{j}$ is strictly positive. We now define the public effect $P_{t}^{j}$, which depends on the shares of agents $x_{t}, z_{t} \in[0,1]$ adopting strategy $\boldsymbol{A}$ at time $t$ in regions $N$ and $S$, respectively. We also differentiate between domestic and foreign effects of the adaptation/mitigation technology, respectively defined as $d^{j}$ and $f^{j}$. The former describes the impact on a local environmental indicator of same-region adopters, whereas the latter 
describes the impact of cross-region adopters. For the sake of simplicity, we assume that the public effects are determined by linear functions:

$$
\begin{gathered}
P_{t}^{N}:=-d^{N} \cdot x_{t}-f^{N} \cdot z_{t} \\
P_{t}^{S}:=-f^{S} \cdot x_{t}-d^{S} \cdot z_{t}
\end{gathered}
$$

where parameters $d^{j}$ and $f^{j}$ measure the domestic and the foreign public effects, respectively, for region $j=N, S$. They represent the public impact of adoption by all agents on the local environmental indicator of region $j$. In other terms, $d^{j}$ and $f^{j}$ represent the externalities embedded in adopting strategy $\boldsymbol{A}$. These externalities might either depend on technological factors (as in solar engineering) and/or on policies regulating them (e.g. emissions standards). Since the analysis of these factors are beyond the scope of the present paper, we assume them to be exogenously determined. Domestic effects $d^{j}$ are caused by agents in region $j$ and worsen the quality of their own local environmental indicator, whereas foreign effects $f^{j}$ affect the local environmental indicator of region $j$ but are caused by agents in the other region. We do not apply any sign restriction on the public effects, so that externalities of adoption of the environmental adaptation technology may take either sign. When a public effect $P^{j}$ is positive, adoption of strategy $\boldsymbol{A}$ by an agent carries part of its benefits over to other agents. This case qualifies as a mitigation case, in which an agent is working for the cooperative improvement of environmental quality, or equivalently towards the abatement of pollution. By contrast, when the public effect is negative, an agent adopting strategy $\boldsymbol{A}$ is actually benefiting herself by worsening environmental quality for others. From the concavity of (1), we may add that a negative public effect affects relatively more (reduces well-being by a higher amount) the agents who are not adopting the environment enhancing strategy $\boldsymbol{A}$. By the definition provided by Barnett and O'Neill (2010), this is a case of maladaptation.

In order to study the dynamics of this system, we now describe the way in which the share of agents adopting strategy $\boldsymbol{A}$ in either country varies. We assume that if the difference in well-being $\Delta \Pi^{j}=\Pi_{A}^{j}-\Pi_{N A}^{j}$ between strategy $\boldsymbol{A}$ and strategy $\boldsymbol{N A}$ is positive for region $j$, then the share of agents adopting the technology (either a maladaptive or a mitigation one) in that region will increase, since it provides higher payoffs. The opposite holds if the payoff difference is negative. Finally, if the payoff difference equals zero, economic agents are indifferent between adopting or not adopting the technology, so that the population shares of agents adopting the technology keeps constant over time. Therefore, we have that:

$$
\Delta \Pi^{N}\left(x_{t}, z_{t}\right) \gtreqless 0 \Rightarrow \dot{x} \gtreqless 0 \quad \Delta \Pi^{S}\left(x_{t}, z_{t}\right) \gtreqless 0 \Rightarrow \dot{z} \gtreqless 0
$$

where $\dot{x}$ and $\dot{z}$ are the time derivatives of $x_{t}$ and $z_{t}$, respectively. Hence, in each region the payoff difference $\Delta \Pi^{j}\left(x_{t}, z_{t}\right)$ in $N$ and $\Delta \Pi^{S}\left(x_{t}, z_{t}\right)$ in $S$ has the same sign as the time derivative of the population share that adopts the environmental adaptation technology in that region. Referring to the well-being definition (1), we may derive the payoff difference $\Delta \Pi^{j}$ :

$$
\Delta \Pi^{j}\left(x_{t}, z_{t}\right)=\Pi_{A}^{j}\left(x_{t}, z_{t}\right)-\Pi_{N A}^{j}\left(x_{t}, z_{t}\right)=\ln \frac{\bar{E}^{j}+p^{j}+P_{t}^{j}}{\bar{E}^{j}+P_{t}^{j}}-C^{j}
$$

We assume that the dynamics of $x_{t}$ and $z_{t}$ is given by the so-called "replicator dynamics"(see e.g. Weibull 1995): 


$$
\left\{\begin{array}{c}
\dot{x}=x(1-x) \Delta \Pi^{N}(x, z) \\
\dot{z}=z(1-z) \Delta \Pi^{S}(x, z)
\end{array}\right.
$$

where we omitted the temporal subscript $t$ to improve readability. Dynamics (5) describes an adaptive process based on an imitation mechanism: every period $t$, a (very) small fraction of the population changes its strategy adopting the more remunerative one. Differently from the "classical" contexts where replicator dynamics are introduced (in which economic agents are pairwise randomly matched), here the well-being of each agent depends on the technological choice by all agents, in both regions, and at the same instant; that is, we analyse a population game. Replicator dynamics may be generated by several learning mechanisms in a random matching context (see e.g. Börgers and Sarin 1997; Schlag 1998); however, rationales for such dynamics can be found also in our context (see e.g. Sacco 1994). Sethi and Somanathan (1996) propose an application of replicator equations in a context similar to ours.

\subsection{Basic Mathematical Results}

As the shares of agents adopting strategy $\boldsymbol{A}$ are defined in the interval $[0,1]$, the dynamic system (5) is defined in the square $\boldsymbol{Q}$ :

$$
Q=\{(x, z): 0 \leq x \leq 1,0 \leq z \leq 1\} .
$$

We will henceforth denote with $Q_{x=0}$ the side of $\boldsymbol{Q}$ along which $x=0$, and with $Q_{x=1}$ the side along which $x=1$. Similar interpretations apply to $Q_{z=0}$ and $Q_{z=1}$. All sides of this square are invariant; in other terms, if the pair $(x, z)$ initially lies on one of the sides, then the whole correspondent trajectory also lies on that side.

Note that the states $\{(x, z)=(0,0),(0,1),(1,0),(1,1)\}$ are always stationary states of the dynamic system (5). In such states, only one strategy (either $\boldsymbol{A}$ or $\boldsymbol{N A}$ ) is played in each region. Other stationary states are the points of intersection between the interior of the sides $Q_{x=0}, Q_{x=1}$ (where $\dot{x}=0$ ) and the locus $\Delta \Pi^{S}(x, z)=0$ (where $\dot{z}=0$ ) and the points of intersection between the interior of sides $Q_{z=0}, Q_{z=1}$ (where $\dot{z}=0$ ) and the locus $\Delta \Pi^{N}(x, z)=0$ (where $\dot{x}=0$ ). In such stationary states, there is a region (either $S$ or $N$ ) where both available strategies are played by a positive share of agents, while in the other region all agents choose the same strategy. In addition, the point in the interior of $\boldsymbol{Q}$ where the loci $\Delta \Pi^{N}(x, z)=0$ and $\Delta \Pi^{S}(x, z)=0$ meet are other possible stationary states. In such points, both strategies are adopted by a positive share of agents in both regions.

Finally, we find that the loci $\dot{x}=0$ and $\dot{z}=0$ in the interior of $Q$ are respectively represented by the lines:

$$
\begin{aligned}
& z=\frac{\bar{E}^{N}}{f^{N}}-\frac{p^{N}}{f^{N}\left(e^{C^{N}}-1\right)}-\frac{d^{N}}{f^{N}} x \\
& z=\frac{\bar{E}^{S}}{d^{S}}-\frac{p^{S}}{d^{S}\left(e^{C^{S}}-1\right)}-\frac{f^{S}}{d^{S}} x
\end{aligned}
$$

where we recall that $e^{C^{j}}-1>0$ since the adoption cost $C^{j}$ is strictly positive. The above lines are obtained by substituting the public effects (2) into the well-being differential (4) and setting it equal to zero. Note that the slope of (6a) and (6b) is negative if the domestic 
effect $d^{j}$ and the foreign effect $f^{j}$ have the same sign, for $j=N, S$ respectively. Furthermore, the slope of (6a) is greater than the slope of (6b) if $\frac{d^{N}}{f^{N}}<\frac{f^{s}}{d^{s}}$. Finally, we note that $\Delta \Pi^{N}(x, z)$ is positive (i.e. $\dot{x}>0$ ) above (6a) if $f^{N}>0$ (vice versa if $f^{N}<0$ ) and that $\Delta \Pi^{S}(x, z)$ is positive (i.e. $\dot{z}>0$ ) above (6b) if $d^{S}>0$ (vice versa if $d^{S}<0$ ). Since both (6a) and (6b) are straight lines, there generally ${ }^{3}$ exists at most one stationary state in the interior of each side of $\boldsymbol{Q}$ and at most one in the interior of $\boldsymbol{Q}$. Consequently, by recalling that all vertices are stationary states, as well, the highest number of stationary states that can be generally observed is nine (four vertices, four points on the sides, and an internal point).

Let us now outline the possible scenarios the system may reach when the adaptation/ mitigation technology is characterised by:

(1) Negative public effects towards all agents. In such a context, the technology is maladaptive, i.e. it is such that it lowers the environmental quality for all. Formally, this maladaptation technology has both a domestic and a foreign negative public effect. One common example of such a technology in the literature is air conditioning: it provides the person with an improvement of her environmental quality at the cost of a small deterioration of the environmental quality (and energy security) for all other people (Deschênes and Greenstone 2011; Lundgren and Kjellstrom 2013). From an analytical perspective, this translates into all public effect parameters being strictly positive: $d^{N}$, $d^{S}, f^{S}, f^{N}>0$.

(2) Positive public effects towards all agents. In such a context, the technology increases the environmental quality for all (mitigation). This mitigation technology has both a domestic and a foreign positive public effect, and this translates into all public effect parameters being strictly negative: $d^{N}, d^{S}, f^{S}, f^{N}<0$.

Other relevant cases could be investigated, yet we restrain the analysis to these two cases for the sake of parsimony.

\section{Technologies with Negative Public Effects}

In this section, we analyse scenario (1), that is the case of a maladaptation technology. In this context, the following inequalities hold:

$$
\begin{gathered}
\frac{\partial \Delta \Pi^{N}(x, z)}{\partial x}=\frac{p^{N} d^{N}}{\left(\bar{E}^{N}+P^{N}\right)\left(\bar{E}^{N}+p^{N}+P^{N}\right)}>0 \\
\frac{\partial \Delta \Pi^{S}(x, z)}{\partial z}=\frac{p^{S} d^{S}}{\left(\bar{E}^{S}+P^{S}\right)\left(\bar{E}^{S}+p^{S}+P^{S}\right)}>0
\end{gathered}
$$

Inequalities (7a) and (7b) indicate that the well-being differential of adopting strategy $\boldsymbol{A}$ increases with the share of adopters. The higher is the proportion of agents adopting it in

\footnotetext{
3 In the unlikely circumstance that lines (6a) and (6b) have the same slope and the same intercept, the two lines completely overlap and all their points in the interior of $\boldsymbol{Q}$ are stationary states.
} 
either group, the higher is the incentive for others to do the same. To fix ideas, we can think to air conditioning. As the additional production of energy demanded by the air conditioning systems of other agents increases the heat in the region, even more agents are incentivised to adopt the maladaptive technology. In this scenario, strategy $\boldsymbol{A}$ yields the highest payoffs when the majority of agents adopts it. As we will see, such a context favours the emergence of self-reinforcing processes that lead agents to adopt only one strategy, in each region $S$ and $N$.

\subsection{Dynamic Regimes}

First of all, we note that if $d^{N}, d^{S}, f^{S}, f^{N}>0$, then both lines (6a) and (6b), along which $\dot{x}=0$ and $\dot{z}=0$, respectively, have negative slope. Above these lines, we have that the share of agents adopting strategy $\boldsymbol{A}$ increases. In particular, $\dot{x}>0$ above line (6a) and $\dot{z}>0$ above line (6b), whereas the reverse occurs below these lines. This is very informative with respect to the behaviour of agents: for a higher value of $x, z$ must be lower in order for agents in either region to be indifferent to the maladaptation technology, or else they would prefer to adopt strategy $\boldsymbol{A}$. From another perspective, for a given point $(x, z)$ which lies on either line (6a) or (6b), a translation (i.e. shift) to the right would make $\boldsymbol{A}$ the preferred strategy in region $N$ or $S$ (or both), respectively. Finally, we note that lines (6a) and (6b) move downwards if the autonomous environmental quality for region $N$ or $S$ is lower. For sufficiently low values of $\bar{E}^{N}$ and $\vec{E} S$, we have that $\dot{x}>0$ and $\dot{z}>0$, respectively, for all points in $\boldsymbol{Q}$. The reverse applies when $\bar{E}^{N}$ and $\bar{E}^{S}$ are sufficiently high.

The following proposition characterises the dynamics of the system when $d^{N}, d^{S}, f^{S}, f^{N}$ $>0$.

Proposition 1 Under the assumption that $d^{N}, d^{S}, f^{S}, f^{N}>0$, the system (5) has the following features:

(a) Every trajectory of the system approaches a stationary state.

(b) Only the vertices of $Q$, i.e. the stationary states $(0,0),(0,1),(1,0),(1,1)$, can be attractive.

Proof See "Mathematical appendix A".

\subsection{Stability Properties of the Vertices}

The analysis in the Mathematical appendix B allows us to illustrate the stability properties of the stationary states $(0,0),(0,1),(1,0),(1,1)$.

\subsubsection{Stability of the Stationary State $(0,0)$}

In this stationary state, no agent adopts the technology. In order for this non-adoption scenario to be attractive, it must be individually convenient to adopt strategy $N \boldsymbol{A}$ in both regions. In order for this to hold, the following condition must be satisfied: 


$$
\bar{E}^{j}>\frac{p^{j}}{e^{C^{j}}-1} \quad \text { with } j=N, S
$$

To the right hand side of this inequality we have the ratio of the positive private effect of the technology over a measure of its cost of adoption, so that we may interpret the ratio as the efficiency of the adaptation technology in region $j$. We note that the denominator is strictly positive since $C^{j}>0$. To the left hand side we have the autonomous environmental quality in $j$, which also coincides with the overall environmental quality since no agent is adopting strategy $\boldsymbol{A}(x=0, z=0)$. Condition (8) thus requires that in both regions the efficiency of the technology is lower than the environmental quality. To continue with the example proposed above, mild temperatures (high level of autonomous environmental quality) and costly air conditioning systems hinder their diffusion in the population.

\subsubsection{Stability of the Stationary State $(0,1)$}

This stationary state represents the $S$-adoption scenario, in which all agents in $S$ adopt the technology, while no agent does so in $N$. It is locally attractive if the following conditions hold:

$$
\begin{aligned}
& \bar{E}^{N}-f^{N}>\frac{p^{N}}{e^{C^{N}}-1} \\
& \bar{E}^{S}-d^{S}<\frac{p^{S}}{e^{C^{S}}-1}
\end{aligned}
$$

We note that condition (9) is similar to condition (8) however, in this case, the autonomous environmental quality $\bar{E}^{N}$ is adjusted by the negative public effect $\left(-f^{N}<0\right)$ of the agents in $S$ adopting strategy $\boldsymbol{A}$ (since $z=1$ ). In other terms, in order for the agents in $N$ to be more convenient not to adopt the technology, its efficiency needs to be lower than the overall environmental quality, which includes the public effects of agents in $S$.

According to condition (10), the adoption of the technology by agents in $S$ requires that its efficiency is greater than the environmental quality, which includes the negative domestic public effect $-d^{S}$.

\subsubsection{Stability of the Stationary State $(1,0)$}

This stationary state represents the $N$-adoption scenario and is specular to the previous one, with all agents in $N$ adopting the technology and no agent adopting it in $S$. It is locally attractive if the following conditions hold:

$$
\begin{aligned}
& \bar{E}^{N}-d^{N}<\frac{p^{N}}{e^{C^{N}}-1} \\
& \bar{E}^{S}-f^{S}>\frac{p^{S}}{e^{C^{S}}-1}
\end{aligned}
$$

According to condition (11), in region $N$ the efficiency of strategy $\boldsymbol{A}$ must be greater than the environmental quality adjusted by the domestic (negative) public effect $-d^{N}$. 
Fig. 1 All nine stationary states exist: the vertices are attractors, the ones on the sides are saddles and the internal one is a repeller

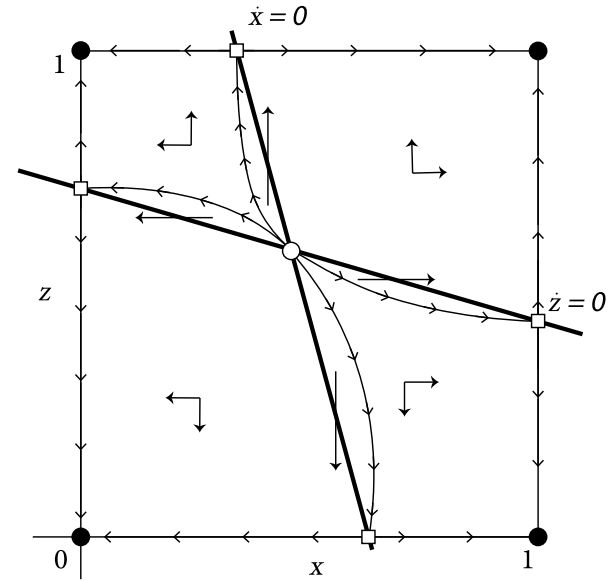

Analogously, according to condition (12), in region $S$ the efficiency of strategy $\boldsymbol{A}$ must be lower than environmental quality, adjusted by the foreign (negative) public effect $-f^{S}$.

\subsubsection{Stability of the Stationary State $(1,1)$}

Finally, the stationary state $(1,1)$ represents a full adoption scenario, in which all agents from both regions adopt the technology. As discussed more in detail later in the paper, this may represent an undesirable outcome. This stationary state is locally attractive if the following conditions hold:

$$
\bar{E}^{j}-\left(d^{j}+f^{j}\right)<\frac{p^{j}}{e^{C^{j}}-1} \quad \text { with } j=N, S
$$

On the left hand side of condition (13) we see that now the environmental quality is affected by both domestic and foreign public effects, since all agents are adopting $\boldsymbol{A}$. The condition requires the efficiency of the technology for both regions to be greater than the environmental quality. A cheap (or a very efficient) technology is easily diffused in a population living with a highly degraded environment.

Finally, we remark that the vertices of $\boldsymbol{Q}$ can be simultaneously attractive, which occurs when the following condition holds:

$$
\frac{p^{j}}{e^{C^{j}}-1}+f^{j}<\bar{E}^{j}<\frac{p^{j}}{e^{C^{j}}-1}+d^{j} \quad \text { with } j=N, S
$$

We note that in order for condition (14) to hold, it is necessary that $f^{j}<d^{j}$ for $j=N, S$. By checking their definitions in (2), we can see that this implies that foreign public effects must be lower than domestic public effects, in both $N$ and $S$. If foreign public effects were stronger than domestic ones in at least one region, then the stationary states $(1,0)$ and $(0,1)$ (respectively, $N$-adoption and $S$-adoption) could not be simultaneously attractive. Indeed, it would not be otherwise convenient for an agent not to adopt strategy $\boldsymbol{A}$ when all agents in the other region are doing so unless foreign public effects were neglectable with respect to domestic ones. 
Fig. 2 In this case, there are three attractors: $(0,0),(0,1)$, $(1,1)$, whereas the other stationary states are either repellers or saddles

Fig. 3 In this case only the stationary states $(0,0)$ and $(1,0)$ are attractors. The stationary state in the interior of the top side of $\boldsymbol{Q}$ is a repeller whereas the one lying in the interior of the botom side is a saddle

Fig. 4 There are two attractors, corresponding to the full adoption $(1,1)$ and the non-adoption $(0,0)$ scenarios. There are also a saddle point on the right hand side and a repeller in the asymmetric state $(0,1)$
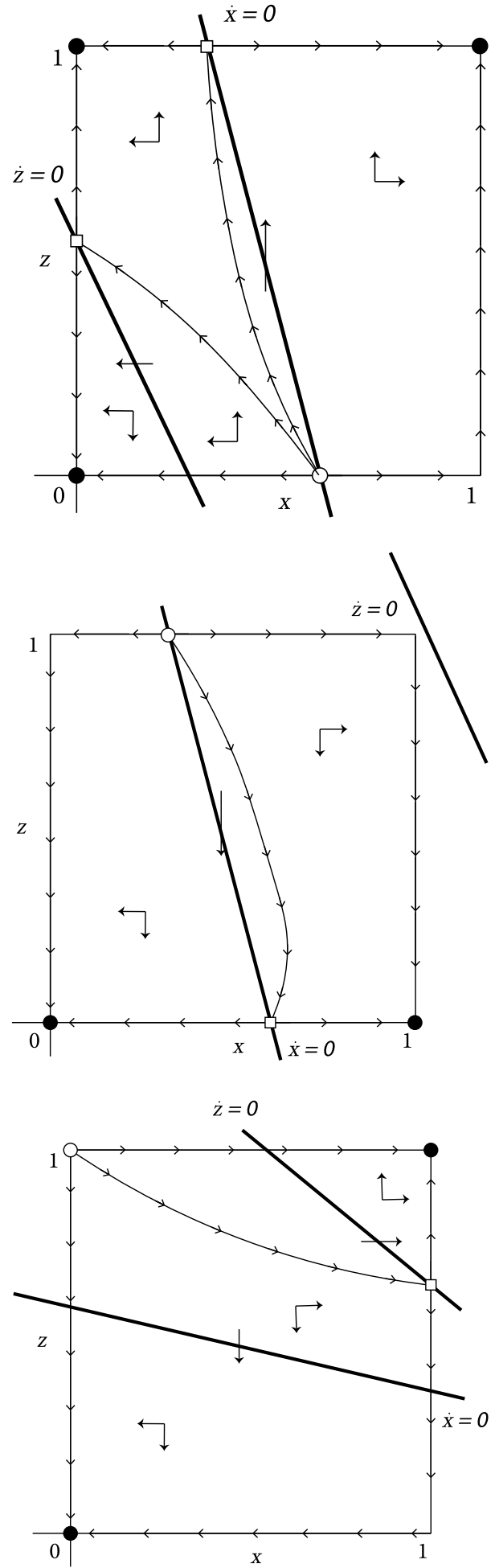
Fig. 5 In this case, the vertices $(0,1)$ and $(1,1)$ are attractors, whereas a repeller lies on the interior of the bottom side of $\boldsymbol{Q}$

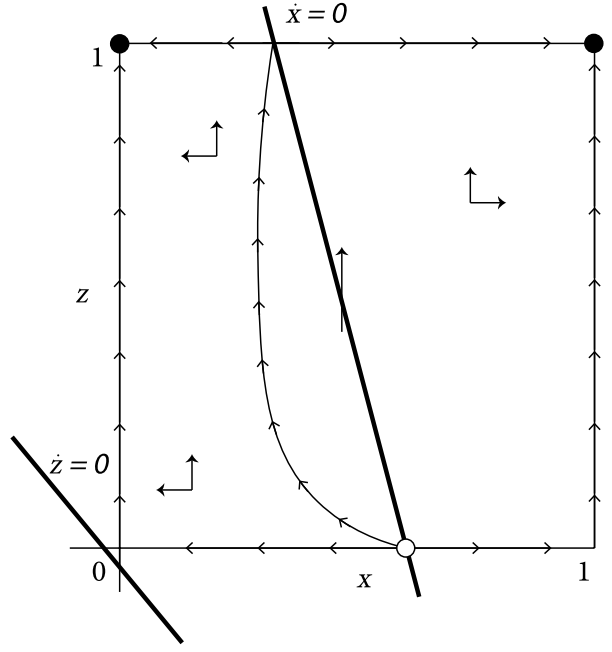

Some examples of multistability are shown in Figs. 1, 2, 3, 4 and 5, where attractive stationary states are represented by full dots $\bullet$, repulsive ones by open dots $\circ$, and saddles by squares $\square$. In all cases graphically represented, agents in each region coordinate on one of the two strategies. The most interesting dynamics of this kind is the one represented in Fig. 1, where condition (14) is satisfied. In this case all vertices of $\boldsymbol{Q}$ are attractive, whereas all other stationary states along the sides of $\boldsymbol{Q}$ are saddle points and the stationary state inside $\boldsymbol{Q}$ is a source. As Fig. 1 shows, almost every trajectory ${ }^{4}$ will lead to a vertex of $\boldsymbol{Q}$, where each region ends up choosing a single strategy (either adopting the environmental maladaptation technology or not). The basins of attraction of the vertices are delimited by the stable manifolds of the saddle point in the interior of the sides of $\boldsymbol{Q}$.

\subsection{Well-Being Analysis}

We now examine the average level of well-being in the two regions when all public effects are negative, i.e. the coefficients are positive: $d^{N}, d^{S}, f^{S}, f^{N}>0$. The average level of wellbeing in $N$ and in $S$ is equal to the weighted average of the well-being of agents adopting strategy $\boldsymbol{A}$ and the well-being of agents adopting $\boldsymbol{N A}$, where the weights are given by share of adopters in the region. Formally, we have that:

$$
\begin{gathered}
\widetilde{\Pi}^{N}(x, z):=x \cdot \Pi_{A}^{N}(x, z)+(1-x) \cdot \Pi_{N A}^{N}(x, z) \\
\widetilde{\Pi}^{S}(x, z):=z \cdot \Pi_{A}^{S}(x, z)+(1-z) \cdot \Pi_{N A}^{S}(x, z)
\end{gathered}
$$

so that $\widetilde{\Pi}^{N}(0, z)=\Pi_{N A}^{N}(0, z)$ represents the average well-being in $N$ when no agent is adopting $\boldsymbol{A}$ in this region, whereas $\widetilde{\Pi}^{N}(1, z)=\Pi_{A}^{N}(1, z)$ represents the opposite case. The interpretation is analogous for region $S$. The following proposition applies:

$\overline{4}$ The stable branches of the saddles are exceptions, as they lead the system toward the saddle points. 
Fig. 6 There exist a global attractor, corresponding to the full adoption scenario $(1,1)$. There is also a repeller in the interior of the bottom side of $\boldsymbol{Q}$

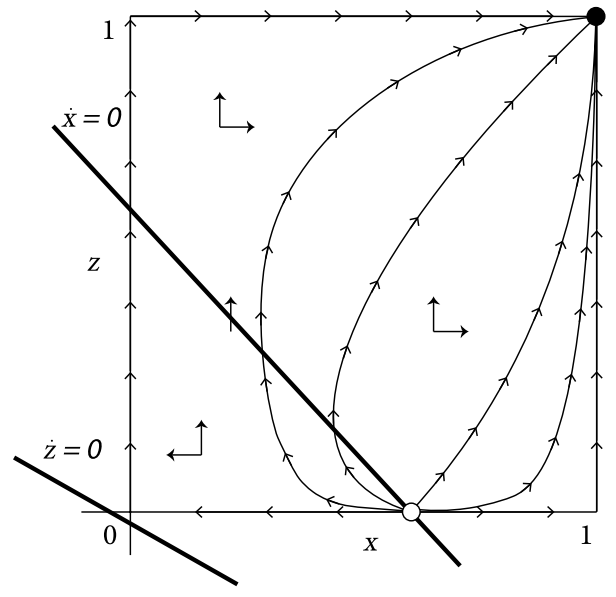

Proposition 2 If $d^{N}, d^{S}, f^{S}, f^{N}>0$, then:

(a) for agents in $N$, the non-adoption stationary state $(0,0)$ Pareto-dominates all other stationary states in $Q$, when they exist, with $0 \leq x<1$ and $0 \leq z \leq 1$. Equivalently, $\widetilde{\Pi}^{N}(0,0)>\widetilde{\Pi}^{N}(x, z)$ for every $(x, z) \neq(0,0)$ with $x$ and $z$ such that $0 \leq x<1$ and $0 \leq z \leq 1$.

(b) for agents in $S$, the non-adoption stationary state $(0,0)$ Pareto-dominates all other stationary states in $Q$, when they exist, with $0 \leq x \leq 1$ and $0 \leq z<1$. Equivalently, $\widetilde{\Pi}^{S}(0,0)>\widetilde{\Pi}^{S}(x, z)$ for every $(x, z) \neq(0,0)$ with $x$ and $z$ such that $0 \leq x \leq 1$ and $0 \leq z<1$.

(c) for agents in both regions $N$ and $S$, the non-adaptation stationary state $(0,0)$ Paretodominates all the other stationary states (among these, also the full adoption stationary state $(1,1))$, when the efficiency of strategy $\boldsymbol{A}$, net of domestic public effects, is lower than local autonomous environmental quality. Equivalently, $\widetilde{\Pi}^{j}(0,0)>\widetilde{\Pi}^{j}(x, z)$ for every $(x, z) \neq(0,0)$ when $\bar{E}^{j}>\frac{p^{j}-d^{j}}{e^{c j}-1}$, with $j=N, S$.

Proof See Mathematical appendix A

By the above proposition and by virtue of Sect. 3.2, it is easy to check that when the stationary state $(0,0)$ is locally attractive, then it Pareto-dominates all others. Furthermore, the non-adoption stationary state $(0,0)$ may Pareto-dominate the stationary state $(1,1)$ (in both regions) even if $(1,1)$ is the only attractive stationary state (see Fig. 6), provided that $\bar{E}^{N}$ and $\bar{E}^{S}$ are sufficiently high. In such case, the adoption of maladaptation technologies in both regions reduces the well-being of agents as system moves from the repulsive non-adoption state $(0,0)$ to the attractive full adoption state $(1,1)$. One could also check that if $(0,0)$ does not Pareto-dominate all other stationary states (in both $N$ and $S$ ), then the dynamics (5) is trivial, i.e. $\dot{x}$ and $\dot{z}$ are always positive in $\boldsymbol{Q}$. In such case, the stationary state $(1,1)$ is globally attractive and Pareto-dominates any other possible state $(x, z)$ in $N$ and $S$. 
Remark From the well-being analysis above, in the context represented in Fig. 1, every agent, from each region, achieves its highest level of well-being in $(0,0)$. Therefore, only one of the four attractive vertices yields the maximum level of well-being. Furthermore, the lowest level of well-being is achieved in $(1,1)$, whereas intermediate levels are reached in $(0,1)$ and $(1,0)$.

\subsection{Environmental Dumping}

At the centre of debates of both environmental and development economists, environmental dumping is the phenomenon for which an economic activity in a more developed country results in the disproportionate degradation of the environment of a less developed country. Some scholars even argued that policies targeted to improve environmental quality in more developed countries lead to increased pollution in less developed ones. For instance, scholars investigating the Pollution Haven Effect ${ }^{5}$ maintain that carbon taxes and stricter environmental regulation are a push factor for firms, which offshore to less developed countries with laxer environmental regulation. Opponents of this theory argue that international trade and offshoring incentivise less developed countries to raise their environmental standards and thus help tackling the problem of environmental degradation. The analysis of the North American Free Trade Agreement performed by Gallagher (2000) seems to partly support both claims: Mexican firms reduced their emission intensity following the agreement, yet overall emissions increased due to the relatively lower Mexican standards with respect to the US ones. Since $\mathrm{CO}_{2}$ emissions are a public bad (their negative effects affect the whole world), this increased pollution might have damaged more developed countries, as well.

We here investigate this hypothesis, for which shifting environmental burden from one country to the other might worsen the well-being of all agents. More precisely, our model allows to study the adoption dynamics of an environmental maladaptation technology with negative public effects and which asymmetrically degrades the environmental quality indicator of one of the two regions. We here discuss what happens when an exogenous factor (e.g. a new policy) decreases domestic effect $d^{N}$ in $N$, whereas it raises the foreign effect $f^{S}$ in $S$. This is the case of a green tax or policy in the more developed region which decreases the domestic effect on the local resource but increases the foreign effect on the resource of the other region, further degrading it. By recalling the payoff differentials (4), we may perform a simple comparative dynamics analysis, noting that a smaller value of $d^{N}$ improves the environmental quality in $N$ and decreases the well-being differential of adopters of the maladaptation technology. This leads to a reduction in the share of adopters in $N$ and the ensuing reduction in environmental degradation. However, an increase in the foreign effect $f^{S}$ on the local environmental indicator of $S$ increases the payoff differential for adopters, incentivising more agents to adopt the maladaptation technology. A higher share of adopters in $S$, i.e. a greater $z$, would then increase the public effect for agents in $N$. The overall well-being effects for agents in $N$ cannot be assessed a priori. If the reduction in the domestic effect $d^{N}$ is sufficiently large with respect to the increase in $f^{S}$, it might counterbalance the additional degradation deriving from more adopters in the $S$ region, who emit the foreign effect $f^{N}$ affecting the environmental quality in region $N$. Vice versa, if the domestic

\footnotetext{
5 See Copeland and Scott Taylor (2004) for a definition of the concept and its differences with the slightly similar Pollution Haven Hypothesis.
} 
effect is weaker with respect to the increased adoption induced in the foreign region, then the well-being of $N$ decreases as a consequence of the exogenous change.

A graphical illustration is provided by Figs. 3 and 4 . In the former figure, only the Pareto-dominant non adoption state $(0,0)$ and the $\mathrm{N}$-adoption state $(1,0)$ are attractive. However, a green policy affecting the value of $d^{N}$ and $f^{S}$ may cause the stationary state $(1,0)$ to lose attractiveness and the Pareto-dominated state $(1,1)$ to become attractive (see Sect. 3.2), as represented in the latter figure. This implies that, depending on the initial condition, a green policy as previously described may push the system towards a Paretodominated state. This analysis highlights that environmental policies in a more developed region may have either a positive or a negative effect for its agents, depending on the feedback effects from agents in the less developed region.

\section{Technologies with Positive Public Effects}

We now study the case in which all public effects of the environmental adaptation technology are positive, that is: $d^{N}, d^{S}, f^{S}, f^{N}<0$. This case describes the adoption dynamics of a mitigation technology, which thus improves environmental quality for all agents. An instance relating to household mitigation strategies is home insulation, as it reduces the energy demand of the residents thus also stimulating lower (polluting) production (see Gupta and Gregg 2012; Hallegatte 2009, for other instances of adaptation technologies with mitigation features). If we think of the agents as firms, instances of such technologies might be the installation of a water treatment plant on a common water basin or, equivalently, of a technology which reduces emissions or waste water usage. Other examples might draw from businesses dealing with the management of common environmental resources, such as fisheries or forestries (Olson 1965).

Analytically, the opposite of inequalities (7a) and (7b) hold, that is:

$$
\begin{gathered}
\frac{\partial \Delta \Pi^{N}(x, z)}{\partial x}=\frac{p^{N} d^{N}}{\left(\bar{E}^{N}+P^{N}\right)\left(\bar{E}^{N}+p^{N}+P^{N}\right)}<0 \\
\frac{\partial \Delta \Pi^{S}(x, z)}{\partial z}=\frac{p^{S} d^{S}}{\left(\bar{E}^{S}+P^{S}\right)\left(\bar{E}^{S}+p^{S}+P^{S}\right)}<0
\end{gathered}
$$

Inequalities (17a) and (17b) describe payoff configurations of strategies $\boldsymbol{A}$ and $\boldsymbol{N A}$ in $N$ and $S$, respectively, similar to those of the "elitist" narratives in (Antoci et al. 2018). Since the well-being differential of adopting strategy $\boldsymbol{A}$ decreases with the share of adopters, strategy $\boldsymbol{A}$ yields the highest payoffs when only a minority of agents adopts it. As strategy $\boldsymbol{A}$ diffuses, so the incentive to adopt it decreases, to the point that agents become indifferent toward the technology. As we will see, the presence of such a property in a region is necessary in order to have coexistence of strategies in such a region.

\subsection{Dynamic Regimes}

We first note that if $d^{N}, d^{S}, f^{S}, f^{N}<0$, both the straight lines (6a) (where $\dot{x}=0$ ) and (6b) (where $\dot{z}=0$ ) have negative slope. Differently from the case with negative public effects, 
Fig. 7 The non-adoption stationary state $(0,0)$ is globally attractive, whereas the full adoption one $(1,1)$ is repulsive

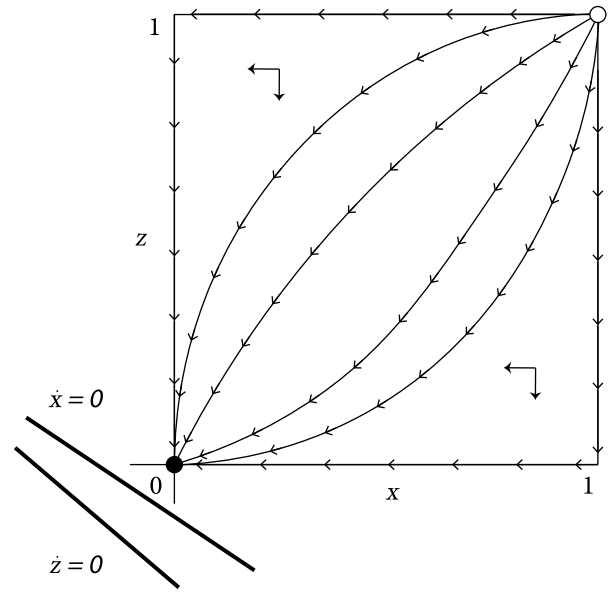

in this case $\dot{x}>0$ below line (6a), whereas $\dot{x}<0$ above it. Analogously, $\dot{z}>0$ below line (6b), whereas $\dot{z}<0$ above it. In contrast to the previous case, now the adoption dynamics is not self-reinforcing: more specifically, the incentive to adopt the environmental mitigation technology decreases if the share of agents adopting the technology in either group increases. This is the well known free riding problem, for which agents are not willing to contribute to a public good and would rather benefit from the contributions of others without paying the cost of their own contribution. In addition, the concavity of the well-being function with respect to the environmental quality accentuates the effect, as it makes any further improvement of the environment less desirable. Since the returns from the mitigation technology decrease with the share of adopters while the cost is constant, we may see why this context favours coexistence between strategies $\boldsymbol{A}$ and $\boldsymbol{N A}$. Indeed, as more and more agents adopt the mitigation technology, the well-being differential of such strategy falls to zero, allowing for a stationary state in which in the same region there are agents adopting strategy $\boldsymbol{A}$ and agents adopting $\boldsymbol{N A}$. In the common water basin example, installing (or contributing to the installation of) a new water treatment plant is less useful for a firm if the quality of the water is already guaranteed by the presence of treatment plants funded by other firms. In addition, we remark that if the autonomous environmental quality $\bar{E}$ is sufficiently high in $N$ and $S$, then the well-being differential is always negative, i.e. $\dot{x}<0$ and $\dot{z}<0$, leading agents to drop the mitigation technology and shift from $\boldsymbol{A}$ to $\boldsymbol{N A}$. In this case, the autonomous level of environmental quality is so high that no agent finds it convenient to increase it further by an amount equal to the private effect $p^{j}$, with $j=0,1$. This might also be due to the inefficiency of the mitigation technology (a low value of $\left.\frac{p^{j}}{e^{c^{j}}-1}\right)$. In formal terms, we may say that lines (6a) and (6b) move downwards if the autonomous environmental quality for region $N$ or $S$ is higher. For sufficiently high values of $\bar{E}^{N}$ and $\bar{E}^{S}$ or for sufficiently low values of $p^{N}$ and $p^{S}$, we have that $\dot{x}<0$ and $\dot{z}<0$, respectively, hold for every value of $x$ and $z$. The reverse applies when $\bar{E}^{N}$ and $\bar{E}^{S}$ are sufficiently low or $p^{N}$ and $p^{S}$ sufficiently high.

We find that the following proposition characterises the adoption dynamics when: $d^{N}$, $d^{S}, f^{S}, f^{N}<0$. 
Proposition 3 Under the assumption that $d^{N}, d^{S}, f^{S}, f^{N}<0$, the system (5) has the following features:

(a) Every trajectory of the system approaches a stationary state.

(b) When the stationary state $(0,0)$ is attractive (see Sect. 3.2), then it is globally attractive, i.e. there is no other attractive stationary state (see Fig.7).

(c) When the stationary state $(1,1)$ is attractive (see Sect. 3.2), then it is globally attractive (see Fig. 8).

(d) If there is no stationary state in the interior of $Q$, then there exists only one attractive stationary state in the boundary of $Q$; it may either be one of the vertices or lie on the interior of the edges of $\boldsymbol{Q}$.

(e) If $d^{N} d^{S}-f^{N} f^{S}>0$, i.e. the domestic effects are larger than the foreign ones, the stationary state in the interior of $\boldsymbol{Q}$ (in which both strategies are played in both regions) is globally attractive, when it exists (see Fig. 9).

(f) If $d^{N} d^{S}-f^{N} f^{S}<0$, i.e. the domestic effects are smaller than the foreign ones, the stationary state in the interior of $\boldsymbol{Q}$ is a saddle point, when it exists. In addition, there exist two attractive stationary states lying in the edges of $Q$ : they may be the vertices $(0,1)$ and $(1,0)$ or lie in the interior of the edges $\boldsymbol{Q}$ (see Figs.10, 11, 12 and 13).

(g) If $p^{N}=p^{S}=0$ (i.e. the private effect of strategy $\boldsymbol{A}$ is 0 in both regions), then non-adoption is individually convenient for all agents: $\Pi_{N A}^{N}>\Pi_{A}^{N}$ and $\Pi_{N A}^{S}>\Pi_{A}^{S}$, whatever the values of $x$ and $z$ are. This implies that $\dot{x}<0$ and $\dot{z}<0$ always hold and consequently $(0,0)$ is globally attractive (the classical free-riding problem arises for public goods provision).

Proof See Mathematical appendix A

Fig. 8 The full adoption stationary state $(1,1)$ is globally attractive, whereas the non-adoption one $(0,0)$ is repulsive

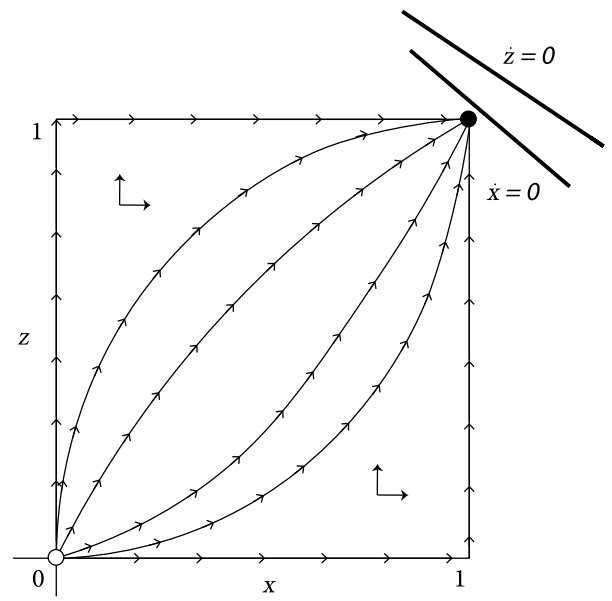


Fig. 9 The internal stationary state is an attractor. There are also three saddles on the sides and three repellers on the vertices $(0,0),(1,0),(1,1)$

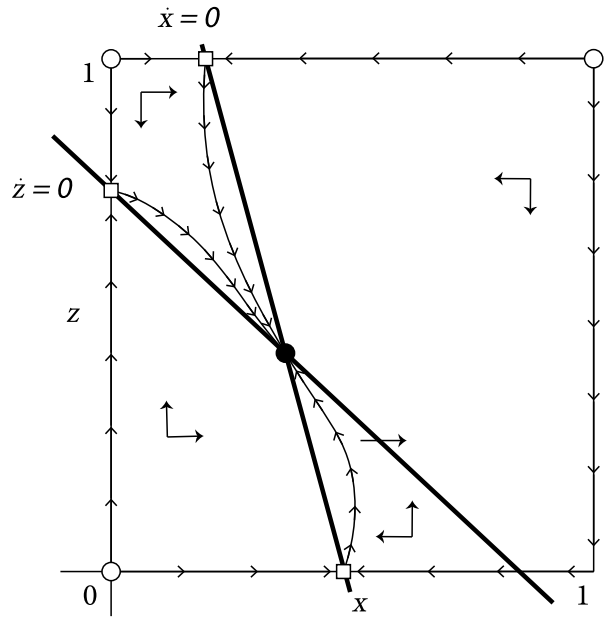

Fig. 10 The internal stationary state is a saddle, whereas the asymmetric states $(0,1)$ and $(1,0)$ are attractors. The nonadoption state $(0,0)$ and the full adoption one $(1,1)$ are repellers

Fig. 11 The internal staationary state is a saddle and both the non-adoption $(0,0)$ and the full adoption $(1,1)$ states are repellers. Two attractors lie on the interiors of the bottom side and of the top side of $\boldsymbol{Q}$
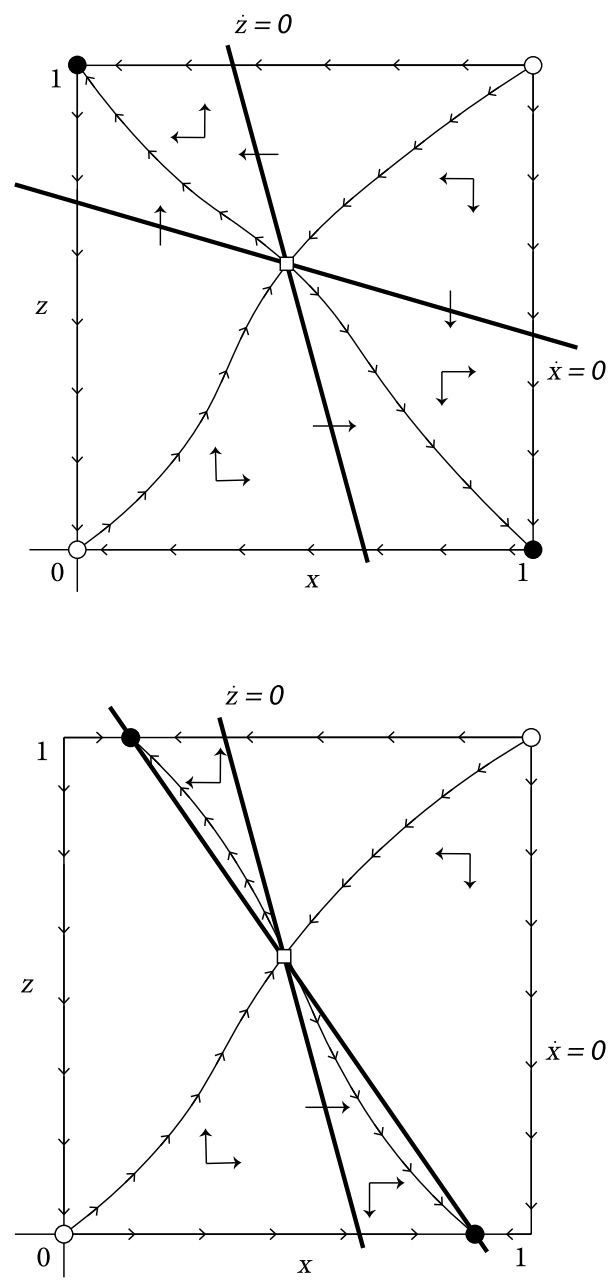
Fig. 12 The internal stationary state is a saddle and both the non-adoption $(0,0)$ and the full adoption $(1,1)$ states are repellers. Two attractors lie on the interiors of the side to the left and of the side to the right of $\boldsymbol{Q}$

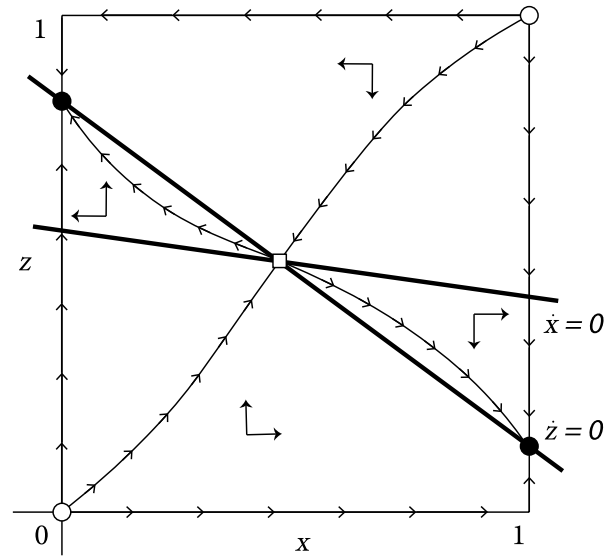

\subsection{Well-Being in the Context with Positive Externalities}

We now examine the average level of well-being in the two regions when all public effects are positive: $d^{N}, d^{S}, f^{S}, f^{N}<0$ (see (15) and (16) in the previous section for a comparison). The following proposition applies:

Proposition 4 Assume $d^{N}, d^{S}, f^{S}, f^{N}<0$. In such context, it holds:

(a) The stationary state $(0,0)$ is Pareto-dominated (in both regions) by any attractive stationary state with $x>0$ and/or $z>0$. When $(0,0)$ is attractive ${ }^{6}$, it may be Paretodominated by other stationary states. ${ }^{7}$

(b) The stationary state $(1,1)$ Pareto dominates (in both regions) any other stationary state when it is attractive (remember that, in such case, no other stationary state can be attractive). Furthermore, even if it is unstable, it Pareto dominates the stationary state in the interior of $\boldsymbol{Q}$, when it exists.

\section{Proof See "Mathematical Appendix A"}

Remark From the well-being analysis above, in the context in which the stationary state $(\bar{x}, \bar{z})$ in the interior of $\boldsymbol{Q}$ is attractive, we have that $(\bar{x}, \bar{z})$ Pareto-dominates $(0,0)$ but is Pareto-dominated by $(1,1)$; however, the latter stationary state cannot be reached because it is not attractive.

These results are reversed with respect to the case with negative public effects. Indeed, in the previous case $(0,0)$ Pareto-dominates all stationary states in most cases, although it is not attractive. The selfish nature of the maladaptation technology leads agents towards

\footnotetext{
${ }^{6}$ As stated in Proposition 3, point $(\mathrm{b})$, in this case $(0,0)$ is globally attractive.

7 This occurs, for instance, when $\frac{p^{N}}{e^{C^{N}}-1}<\bar{E}^{N}<\frac{p^{N}-d^{N}-f^{N}}{e^{e^{N}}-1}$ and $\frac{p^{S}}{e^{C^{S}}-1}<\bar{E}<\frac{p^{S}-d^{S}-f^{S}}{e^{C^{S}}-1}$ hold. Indeed, in this case $(0,0)$ is attractive but is Pareto-dominated by $(1,1)$.
} 
Fig. 13 In this case, one attractor is the asymmetric state $(0,1)$ and another lies on the interior of the right-hand of square $\boldsymbol{Q}$. The internal stationary state is a saddle and both the non-adoption $(0,0)$ and the full adoption $(1,1)$ states are repellers

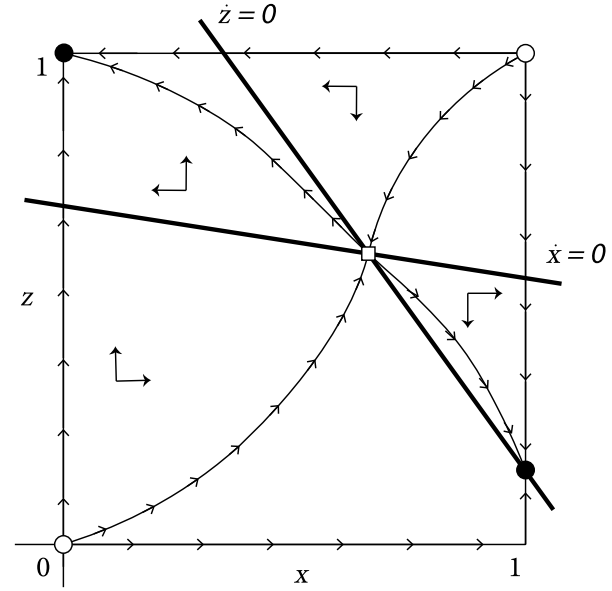

adoption, although it results in a lower level of well-being for all. The technology is thus over-adopted with respect to the Pareto-optimum. With positive public effects, we have that $(0,0)$ is Pareto-dominated by all other stationary states whereas $(1,1)$ Pareto-dominates them when it is attractive. All agents benefit from the mitigation technology adopted by others, but they are less willing to pay its cost as they do not internalise the well-being of others. In this case, the technology is under-adopted, as the full adoption scenario would be the Pareto optimum. This last result is in line with the results by Shogren and Crocker (1991).

\section{Discussion and Conclusions}

In this work, we studied the case of two regions whose agents may adopt an environmental adaptation technology which yields a private benefit to the adopter, while also transferring a negative or positive externality both to agents in the same region and to agents in the other one. We defined same-region externalities as domestic public effects and cross-region externalities as foreign public effects. We excluded altruistic consideration on the part of agents towards either same-region and cross-region agents. In other terms, we assumed that the actions of agents are only driven by self-interest considerations. In addition, we posed no restriction on the initial distribution of the system, so that it might initially lie on a point which is characterised by a positive share of adopters in both regions, even if in that point the dynamics of the system converges to the non-adoption state $(0,0)$. We preferred not to make any assumption on the initial distribution, which could be affected for instance by technological shocks or by early adoption of agents during a learning phase in which the payoff of each strategy is not already known. The model here proposed is very broad, so that a complete analysis of all possible specifications is beyond the scope of this paper. Instead, we focused on two salient characterisations. On the one hand, we analysed the case of a maladaptation technology, whose domestic and foreign public effects are both negative. In this case, an adopter shifts the environmental load to agents from both regions. A common example of this kind of technologies is air conditioning (Lundgren and Kjellstrom 2013). On the other hand, we analysed the case of a mitigation technology, whose domestic 
and foreign public effects are both positive. In this case, each adopter is improving the well-being of agents from both regions. In analogy with the previous example, we may think of home insulation, as it allows each household to reduce both heating and air conditioning, benefiting the environment on a global scale. Our results show that for the maladaptation technology the social optimum is represented by the non-adoption scenario, unless the efficiency of the technology is extremely high (greater than the autonomous level of environmental quality). However, Pareto-dominated states may be reached, because agents do not internalise the externalities of the technology. In this case, we talk of over-adoption of the maladaptation technology. The reverse occurs with a mitigation technology, which would have a full adoption scenario as its Pareto-optimum. However, an intermediate state (in which only some agents are adopters) is typically reached, since the returns on adoption decrease for each additional adopter. Also in this case, agents do not take into account the (positive) externalities of adoption on other agents, this time leading to under-adoption of the technology with respect to the Pareto-dominant state. Finally, under the hypothesis of a maladaptation technology, with negative public effects, we analysed the effects of a green policy which results in environmental dumping. We represented a green policy as one reducing domestic emissions in the more developed region while increasing emissions toward the less developed region. Although it is intuitive that the agents from the less developed region would be worse off in this case, we showed that the implications for agents from the more developed region are not straightforward. Indeed, according to the relative magnitude of the domestic and the foreign effect of the policy, the well-being of agents from the more developed region could decrease as well, with the system reaching a Pareto-dominated state that was not previously attractive.

This last result is particularly interesting, although its plausibility should be verified by further research. Indeed, instances of such negative feedbacks could provide greater insight on the cost-benefit analysis of many maladaptive strategies or policies available to the more developed regions. In addition, further research should try to map the specifications which are not illustrated in this work. Interesting dynamics could arise, for example, if the public effects had different signs according to whether they are domestic or foreign. In particular, a case in which all domestic public effects are null or positive, while all foreign effects are negative would depict a situation in which all adopters shift the environmental burden to foreign agents, although they increase the well-being of same-region individuals. In this case, it is not intuitive which state the system would reach. Another relevant case would be represented by technological differences between the two regions allowing the agents from the more developed region to adopt a mitigation technology, whereas agents in the less developed region could only adopt a maladaptation technology. Well-being analysis could highlight which region is relatively more affected by the negative externalities and which state is more likely to be reached. All similar research directions, focusing on translating real phenomena and dynamics into the model, would provide a fine extension to this work and a contribution to the understanding of the relationship between regions and countries at different stages of development and their environmental quality. 


\section{Appendix A: Proofs of the Propositions in Text}

Proof of Proposition 1 The proof of point (b) is straightforward and follows immediately from the local stability analysis (which can be found in Mathematical appendix B). To prove point (a) we have to show that limit cycles cannot exist (see e.g. Lefschetz 1963, pp. $230 \mathrm{ff})$. This is obviously the case when the internal stationary state $(\bar{x}, \bar{z})$, with $0<\bar{x}, \bar{z}<1$, does not exist or, if it does, is a saddle point. If $(\bar{x}, \bar{z})$ is a source, then $d^{N} d^{S}-f^{S} f^{N}>0$ (see (19b) in appendix B), that is the straight line (6a) (where $\dot{x}=0$ ) crosses from above the straight line (6b) (where $\dot{z}=0$ ). In such case, it is easy to see that the regions in $\boldsymbol{Q}$ where $\dot{x}$ and $\dot{z}$ have the same sign are positively invariant, so that no oscillatory behaviour of trajectories can occur. This implies, by the Poincaré-Bendixson Theorem, that any trajectory starting in $\boldsymbol{Q}$ approaches a stationary state. This concludes the proof of the proposition.

Proof of Proposition 2 To prove point (a) of Proposition 2, we have to show that the average payoff in $N$, evaluated at $(0,0)$, is higher than at any point $(\bar{x}, \bar{z})$ along the line $\Delta \Pi^{N}(x, z)=0$ (where $\dot{x}=0$ ) and along the side $Q_{x=0}$. The average level of well-being in $(0,0)$ is:

$$
\widetilde{\Pi}^{N}(0,0)=\Pi_{N A}^{N}(0,0)=\ln \bar{E}^{N}
$$

Let us now take a point $(\bar{x}, \bar{z}) \in\left\{\Delta \Pi^{N}(x, z)=0\right\}$. We have that both strategies yield the same level of well-being: $\Pi_{A}^{N}(\bar{x}, \bar{z})=\Pi_{N A}^{N}(\bar{x}, \bar{z})$, which implies:

$$
\widetilde{\Pi}^{N}(\bar{x}, \bar{z})=\Pi_{N A}^{N}(\bar{x}, \bar{z})=\ln \left(\bar{E}^{N}-d^{N} \cdot \bar{x}-f^{N} \cdot \bar{z}\right)
$$

Therefore, if $\bar{x}$ and/or $\bar{z}>0$, it follows that: $\widetilde{\Pi}^{N}(0,0)>\widetilde{\Pi}^{N}(\bar{x}, \bar{z})$. This means that the average well-being in the non-adoption state $(0,0)$ is higher than in any stationary state in the interior of $\boldsymbol{Q}$ and in any stationary state in the interior of the sides $Q_{z=h}(h=1,2)$. Furthermore, it is easy to check that $(0,0)$ always Pareto-dominates any stationary state with $z>0$ in the side $Q_{x=0}$. The proof of point (b) can be obtained applying the same arguments.

In order to prove point (c), we now show that, for agents in region $N,(0,0)$ Pareto-dominates any stationary state in the side $Q_{x=1}$ if $\frac{\bar{E}^{N}}{p^{N}-d^{N}}$. It can be easily verified that $(1,0)$ always Pareto-dominates any other stationary state in the side $Q_{x=1}$. Therefore, we simply have to compare well-being in $(0,0)$ with the one in $(1,0)$. By very simple computations, we obtain that, if $\bar{E}^{N}>\frac{p^{N}-d^{N}}{e^{C^{N}}-1}$, then $(0,0)$ Pareto-dominates $(1,0)$. With similar arguments, it is easy to check that $(1,1)$ is Pareto-dominated by all the other stationary states when $\frac{{ }^{N}}{E}>\frac{p^{N}-d^{N}}{e^{C^{N}}-1}$. To prove that analogous results hold for the well-being of region $S$, it suffices to apply the same arguments.

Proof of Proposition 3 The proof of point (b) is straightforward and follows immediately from graphical analysis: if $(0,0)$ is attractive, then it must lie above the straight lines $(6 a)$ and (6b). Consequently, in the interior of $\boldsymbol{Q}$, it holds $\dot{x}<0$ and $\dot{z}<0$, which implies the global attractiveness of $(0,0)$. With similar arguments, point (c) can be proved. In order to prove point (e), it suffices to check that when $d^{N} / f^{N}>f^{S} / d^{S}$, the internal stationary state is locally attractive (see Proposition 6). Graphical analysis then allows to see that no other attractive stationary state can exist. It remains to show that limit cycles cannot exist. To do so, we note that the straight line (6a), along which $\dot{x}=0$, crosses the straight line (6b), along which $\dot{z}=0$, from above. In such case, the regions of $\boldsymbol{Q}$ where $\dot{x}$ and $\dot{z}$ have opposite signs are positively invariant; this implies that no oscillatory behaviour of trajectories may occur and consequently that the internal stationary state is globally attractive by 
the Poincaré-Bendixson Theorem. We now prove point (f): if $d^{N} / f^{N}<f^{S} / d^{S}$, the internal stationary state is a saddle point (see Sect. 3.2); consequently, no limit cycle may exist. Furthermore, we note that the straight line (6a) crosses the straight line (6b) from below. In such case, the regions of $\boldsymbol{Q}$ where $\dot{x}$ and $\dot{z}$ have opposite sign are positively invariant and, in each of these regions, the trajectories approach a stationary state lying on the boundary of $Q$. Finally, the proof of points (a), (d) and (g) is straightforward.

Proof of Proposition 4 To prove point (a) of the proposition, we first consider the average well-being in $N$, which in $(0,0)$ is equal to:

$$
\widetilde{\Pi}^{N}(0,0)=\Pi_{N A}^{N}(0,0)=\ln \bar{E}^{N}
$$

Let us now consider a point $(\bar{x}, \bar{z}) \in Q$. If $(\bar{x}, \bar{z})$ is a stationary state belonging to the curve $\Delta \Pi^{N}(x, z)=0$, then it holds that $\Pi_{A}^{N}(\bar{x}, \bar{z})=\Pi_{N A}^{N}(\bar{x}, \bar{z})$, and consequently we have:

$$
\widetilde{\Pi}^{N}(\bar{x}, \bar{z})=\Pi_{N A}^{N}(\bar{x}, \bar{z})=\ln \left(\bar{E}^{N}-d^{N} \cdot \bar{x}-f^{N} \cdot \bar{z}\right)
$$

Therefore, since $d^{N}, d^{S}, f^{S}, f^{N}<0$, if either $\bar{x}$ or $\bar{z}>0$, we have that: $\widetilde{\Pi}^{N}(0,0)<\widetilde{\Pi}^{N}(\bar{x}, \bar{z})$. Thus, average payoff in $(0,0)$ is lower than in any stationary state in the interior of $\boldsymbol{Q}$ and in any stationary state in the interior of the sides $Q_{z=k}(k=1,2)$. Furthermore, it is easy to check that $(0,0)$ is always Pareto-dominated by any stationary state in the side $Q_{x=0}$. It remains to prove that $(0,0)$ is Pareto-dominated by any attractive stationary state in

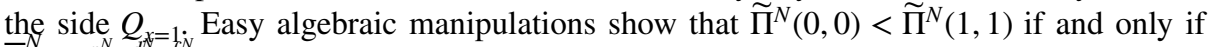
$\bar{E}^{N}<\frac{p^{N}-d^{N}-f^{N}}{e^{C^{N}}-1}$. The latter condition is always satisfied if $(1,1)$ is attractive (see Sect. 3.2). In the same way, it can be checked that $\widetilde{\Pi}^{N}(0,0)<\widetilde{\Pi}^{N}(1,0)$ when $(1,0)$ is attractive. Finally, it is left to prove that $(0,0)$ is Pareto-dominated by any attractive stationary state $(1, \bar{z})$ lying in the interior of $Q_{x=1}$. As already seen above, the well-being in $(0,0)$ is lower than in any stationary state, so that $\widetilde{\Pi}^{N}(0,0)=\Pi_{N A}^{N}(0,0)<\Pi_{N A}^{N}(1, \bar{z})$. Furthermore, we note that if $(1, \bar{z})$ is attractive, then the curve $\Delta \Pi^{N}(x, z)=0$ must lie on the right of it (see Proposition $5)$; consequently, on the left of $\Delta \Pi^{N}(x, z)=0$, it holds that $\Delta \Pi^{N}(x, z)>0$. This implies that $\Pi_{N A}^{N}(1, \bar{z})<\Pi_{A}^{N}(1, \bar{z})$. Therefore, $\widetilde{\Pi}^{N}(0,0)<\widetilde{\Pi}^{N}(1, \bar{z})$, being $\widetilde{\Pi}^{N}(1, \bar{z})=\Pi_{A}^{N}(1, \bar{z})$. The corresponding results for $S$ can be proved following the same steps. To check the remaining part of point (a), we simply have to solve the inequality $\widetilde{\Pi}^{N}(0,0)<\widetilde{\Pi}^{N}(1,1)$ and draw from the stability results in Sect. 3.2 about the stationary state $(0,0)$. The proof of point $(b)$ follows very similar steps.

\section{Appendix B: Stability Properties of the Stationary States}

We here study the stability of the stationary states, in order to understand toward which the system may converge. Indeed, the attractive states are of particular interest, as they are the only limit sets that can actually be reached by the system. We recall that the condition for a 
stationary state to be attractive is that both the eigenvalues of the Jacobian matrix evaluated on it are negative. ${ }^{8}$.

\section{Appendix B.1: Stability Analysis of the Vertices}

In order to assess the stability properties of the vertices of $\boldsymbol{Q}$, we derive the Jacobian matrix of the system (5) evaluated at the stationary state $(x, z)=(i, k), i=0,1$ and $k=0,1$ :

$$
\left(\begin{array}{cc}
(1-2 i) \Delta \Pi^{N}(i, k) & 0 \\
0 & (1-2 k) \Delta \Pi^{S}(i, k)
\end{array}\right)
$$

which has the eigenvalues $(1-2 i) \Delta \Pi^{N}(i, k)$ and $(1-2 k) \Delta \Pi^{S}(i, k)$, in the direction of $\boldsymbol{Q}_{z=\mathbf{0}}$ and $\boldsymbol{Q}_{\boldsymbol{x}=\mathbf{0}}$, respectively.

The analysis of the sign of the eigenvalues allows us to illustrate the stability properties of the stationary states $(0,0),(0,1),(1,0),(1,1)$. It is easy to check that:

In $(0,0)$ both the eigenvalues are strictly negative if:

$$
\bar{E}^{j}>\frac{p^{j}}{e^{C^{j}}-1} \quad \text { with } j=N, S
$$

In $(0,1)$, the eigenvalue in direction of $\boldsymbol{Q}_{z=1}$ is strictly negative if:

$$
\bar{E}^{N}-f^{N}>\frac{p^{N}}{e^{C^{N}}-1}
$$

whereas the eigenvalue in direction of $\boldsymbol{Q}_{x=0}$ is strictly negative if:

$$
\bar{E}^{S}-d^{S}<\frac{p^{S}}{e^{C^{S}}-1}
$$

In $(1,0)$, the eigenvalue in direction of $\boldsymbol{Q}_{z=0}$ is strictly negative if:

$$
\bar{E}^{N}-d^{N}<\frac{p^{N}}{e^{C^{N}}-1}
$$

while the eigenvalue in direction of $\boldsymbol{Q}_{x=1}$ is strictly negative if:

$$
\bar{E}^{S}-f^{S}>\frac{p^{S}}{e^{C^{S}}-1}
$$

Finally, in $(1,1)$ the eigenvalues in direction of $\boldsymbol{Q}_{z=1}$ and $\boldsymbol{Q}_{x=1}$ are strictly negative if:

$$
\bar{E}^{j}-\left(d^{j}+f^{j}\right)<\frac{p^{j}}{e^{C^{j}}-1} \quad \text { with } j=N, S
$$

\footnotetext{
${ }^{8}$ If the eigenvalues are both positive, then the state is repulsive and cannot be reached by system (unless it coincides with its initial condition). If they have opposite signs, instead, the state is a saddle and can only be reached if the initial condition of the system lies on its stable branch.
} 


\section{Appendix B.2: Stability Properties of the Stationary States in the Interior of the Edges of $Q$}

Let us now consider the stability properties of the stationary states belonging to the interior of the edges of the square $Q$, i.e. those where both adoption choices coexist in $N$ while all agents in $S$ play the same strategy or vice versa.

Proposition 5 The Jacobian matrix of the system (5) evaluated at the stationary states in the interior of the edges $Q_{x=h}(h=0,1)$ is:

$$
\left(\begin{array}{cc}
(1-2 h) \Delta \Pi^{N}(h, \bar{z}) & 0 \\
\bar{z}(1-\bar{z}) \frac{\partial \Delta \Pi^{S}(h, \bar{z})}{\partial x} & \bar{z}(1-\bar{z}) \frac{\partial \Delta \Pi^{S}(h, \bar{z})}{\partial z}
\end{array}\right)
$$

where $\bar{z}$ is the value of $z$ at the stationary state, and has the eigenvalues: $\bar{z}(1-\bar{z}) \frac{\partial \Delta \Pi^{S}(h, \bar{z})}{\partial z}$ (in direction of $Q_{x=h}$ ) and $(1-2 h) \Delta \Pi^{N}(h, \bar{z})$ (in direction of the interior of $\boldsymbol{Q}$ ). The Jacobian matrix of the system (5) evaluated at the stationary states in the interior of the edges $Q_{z=h}$ $(h=0,1)$ is:

$$
\left(\begin{array}{cc}
\bar{x}(1-\bar{x}) \frac{\partial \Delta \Pi^{N}(\bar{x}, h)}{\partial x} & \bar{x}(1-\bar{x}) \frac{\partial \Delta \Pi^{N}(\bar{x}, h)}{\partial z} \\
0 & (1-2 h) \Delta \Pi^{S}(\bar{x}, h)
\end{array}\right)
$$

where $\bar{x}$ is the value of $x$ at the stationary state, and has the eigenvalues: $\bar{x}(1-\bar{x}) \frac{\partial \Delta \Pi^{N}(\bar{x}, h)}{\partial x}$ (in direction of $Q_{z=h}$ ) and $(1-2 h) \Delta \Pi^{S}(\bar{x}, h)$ (in direction of the interior of $\boldsymbol{Q}$ ).

We remark that, given a stationary state in an edge $Q_{h=k}, h=x, z$ and $k=0,1$, the sign of its eigenvalue in direction of $Q_{h=k}$ is negative if and only if the stationary states at the extreme of $Q_{h=k}$ which are the vertices of $\boldsymbol{Q}$, have positive eigenvalues in direction of $Q_{h=k}$.

Note that the attractiveness conditions of the stationary states belonging to the interior of the edges of the square $Q$ require that:

$$
\begin{aligned}
\frac{\partial \Delta \Pi^{N}(\bar{x}, i)}{\partial x} & <0 \\
\frac{\partial \Delta \Pi^{S}(i, \bar{z})}{\partial z} & <0
\end{aligned}
$$

Such conditions are never (always) satisfied in the context of technologies with negative (positive) public effects (see formulas (17a) and (17b)). So, in the context where technologies have a negative public effect, the stationary states internal to the edges $Q_{h=k}, h=x, z$ and $k=0,1$, are never attractive (they may be either saddle points or sources).

\section{Appendix B.3: Stability Properties of Stationary States in the Interior of $Q$}

In this subsection we analyse the stability of stationary states in the interior of the square $\boldsymbol{Q}$, in which a positive share of agents adopts each strategy in both regions.

Proposition 6 The Jacobian matrix of the system (5) evaluated at a stationary state $(\bar{x}, \bar{z})$ in the interior of $\boldsymbol{Q}$ (i.e. $0<\bar{x}, \bar{z}<1$ ) is: 


$$
\left(\begin{array}{ll}
\bar{x}(1-\bar{x}) \frac{\partial \Delta \Pi^{N}(\bar{x}, \bar{z})}{\partial x} & \bar{x}(1-\bar{x}) \frac{\partial \Delta \Pi^{N}(\bar{x}, \bar{z})}{\partial z} \\
\bar{z}(1-\bar{z}) \frac{\partial \Delta \Pi^{S}(\bar{x}, \bar{z})}{\partial x} & \bar{z}(1-\bar{z}) \frac{\partial \Delta \Pi^{S}(\bar{x}, \bar{z})}{\partial z}
\end{array}\right)
$$

where the sign of the determinant of $(21 a)$ is equal to the sign of the expression:

$$
d^{N} d^{S}-f^{S} f^{N}
$$

and the trace of (21a) is equal to:

$$
\begin{gathered}
\\
\bar{x}(1-\bar{x}) \frac{\partial \Delta \Pi^{N}(\bar{x}, \bar{z})}{\partial x}+\bar{z}(1-\bar{z}) \frac{\partial \Delta \Pi^{S}(\bar{x}, \bar{z})}{\partial z}= \\
=\bar{x}(1-\bar{x}) \frac{p^{N} d^{N}}{e^{C^{N}}\left(\bar{E}^{N}+P^{N}\right)^{2}}+\bar{z}(1-\bar{z}) \frac{p^{S} d^{S}}{e^{C^{S}\left(\bar{E}^{S}+P^{S}\right)^{2}}}
\end{gathered}
$$

If expression (21b) is strictly negative, then the internal stationary state is a saddle (i.e. it is unstable). If it is positive, then the stationary state may be a repeller or an attractor. In the context in which expression (21b) is strictly positive, the condition $d^{N}, d^{S}>0(<0)$ is a sufficient condition for the repulsiveness (attractiveness) of the internal stationary state (see formula (21c)). Therefore, in the context where technologies have a negative public effect, the internal stationary state is never attractive (it may be either a saddle point or a source).

Funding Open access funding provided by Università degli Studi di Sassari within the CRUI-CARE Agreement. The research of Angelo Antoci was supported by the University of Sassari ("Finanziamento straordinario una tantum per la ricerca 2020"). Giulio Galdi gratefully acknowledges the scholarship received from the Department of International and Political Sciences of the University of Siena for the research activities conducted under the supervision of Simone Borghesi (PSR2017).

Open Access This article is licensed under a Creative Commons Attribution 4.0 International License, which permits use, sharing, adaptation, distribution and reproduction in any medium or format, as long as you give appropriate credit to the original author(s) and the source, provide a link to the Creative Commons licence, and indicate if changes were made. The images or other third party material in this article are included in the article's Creative Commons licence, unless indicated otherwise in a credit line to the material. If material is not included in the article's Creative Commons licence and your intended use is not permitted by statutory regulation or exceeds the permitted use, you will need to obtain permission directly from the copyright holder. To view a copy of this licence, visit http://creativecommons.org/licenses/by/4.0/.

\section{References}

Antoci A, Bellanca N, Galdi G (2018) At the relational crossroads: narrative selection, contamination, biodiversity in trans-local contexts. J Econ Behav Organ 150:98-113

Antoci A, Russu P, Ticci E (2020) Modeling maladaptation in the inequality-environment nexus. J Econ Interact Coord, in press. https://doi.org/10.1007/s11403-020-00301-6

Barnett J, O'Neill S (2010) Maladaptation. Global Environ Change 2:211-213

Bird PJWN (1987) The transferability and depletability of externalities. J Environ Econ Manage 14:54-57

Börgers T, Sarin R (1997) Learning through reinforcement and replicator dynamics. J Econ Theory 77:1-14

Copeland BR, Scott Taylor M (2004) Trade, growth, and the environment. J Econ Literat 42:7-71

Deschênes O, Greenstone M (2011) Climate change, mortality, and adaptation: evidence from annual fluctuations in weather in the US. Am Econ J Appl Econ 3:152-85

Gallagher K (2000) Trade liberalization and industrial pollution in Mexico: lessons of the FTAA, vol 00-07. Tufts University 
Geaun JC (1993) On the shiftable externalities. J Environ Econ Manage 25:30-44

Glotzbach S, Baumgartner S (2012) The relationship between intragenerational and intergenerational ecological justice. Environ Values 21:331-355

Gupta R, Gregg M (2012) Using UK climate change projections to adapt existing English homes for a warming climate. Build Environ 55:20-42

Hallegatte S (2009) Strategies to adapt to an uncertain climate change. Global Environ Change 19:240-247

Hasson R, Löfgren $\AA$, Visser M (2010) Climate change in a public goods game: investment decision in mitigation versus adaptation. Ecol Econ 70:331-338

Heller, WP, Starrett David A (1976) On the nature of externalities. In: Theory and measurement of economic externalities. Elsevier, pp 9-27

IPCC (2014) Climate change 2014: mitigation of climate change. In: Contribution of working group III to the fifth assessment report of the intergovernmental panel on climate change. Cambridge University Press, Cambridge, United Kingdom and New York, NY, USA

IPCC (2018) Global warming of $1.5^{\circ} \mathrm{C}$ : an IPCC special report on the impacts of global warming of $1.5^{\circ} \mathrm{C}$ above pre-industrial levels and related global greenhouse gas emission pathways, in the context of strengthening the global response to the threat of climate change, sustainable development, and efforts to eradicate poverty. Intergovernmental panel on climate change

Lefschetz S (1963) Differential equations: geometric theory, vol 6. Interscience publishers, New York

Lundgren K, Kjellstrom T (2013) Sustainability challenges from climate change and air conditioning use in urban areas. Sustainability 5:3116-3128

Milinski M, Semmann D, Krambeck H-J, Marotzke J (2006) Stabilizing the Earth's climate is not a losing game: supporting evidence from public goods experiments. Proc Nat Acad Sci 103:3994-3998

Milly PCD, Betancourt J, Falkenmark M, Hirsch RM, Kundzewicz ZW, Lettenmaier DP, Stouffer RJ (2008) Stationarity is dead: whither water management? Science 319:573-574

Olson M (1965) Logic of collective action: public goods and the theory of groups, vol 124 of Harvard economic studies. Harvard University Press

Reynolds JL (2019) Solar geoengineering to reduce climate change: a review of governance proposals. Proc Roy Soc A 475:20190255

Rogelj J, Popp A, Calvin KV, Luderer G, Emmerling J, Gernaat D, Fujimori S, Strefler J, Hasegawa T, Marangoni G et al (2018) Scenarios towards limiting global mean temperature increase below $1.5 \mathrm{C}$. Nature Clim Change 8:325-332

Sacco PL (1994) Selection mechanisms in economics. Behav Sci 39:311-325

Schlag KH (1998) Why imitate, and if so, how? A boundedly rational approach to multi-armed bandits. J Econ Theory 78:130-156

Sethi R, Eswaran S (1996) The evolution of social norms in common property resource use. Am Econ Rev 766-788

Shaw D, Shaw R-D (1991) The resistibility and shiftability of depletable externalities. J Environ Econ Manage 20:224-233

Shogren JF, Crocker TD (1991) Cooperative and noncooperative protection against transferable and filterable externalities. Environ Resour Econ 1:195-214

Smith P, Martino D, Cai Z, Gwary D, Janzen H, Kumar P, McCarl B, Ogle S, O'Mara F, Rice C et al (2007) Greenhouse gas mitigation in agriculture. Philos Trans Roy Soc B Biol Sci 363:789-813

Tompkins EL, Neil Adger W, Boyd E, Nicholson-Cole S, Weatherhead K, Arnell N (2010) Observed adaptation to climate change: UK evidence of transition to a well-adapting society. Global Environ Change 20:627-635

UNEP (2019) Frontiers 2018/19 emerging issues of environmental concern. Technical report. United Nation Environment Programme, Nairobi

Wagner G, Weitzman Martin L (2015) Bailing out the planet

Warburton CES et al (2018) Positive time preference and environmental degradation: the effects of world population growth and economic activity on intergenerational equity, 1970-2015. Appl Economet Int Dev 18:5-24

Weibull Jörgen W (1995) Evolutionary game theory. MIT press

Zhang Z, Moore JC, Huisingh D, Zhao Y (2015) Review of geoengineering approaches to mitigating climate change. J Clean Prod 103:898-907

Publisher's Note Springer Nature remains neutral with regard to jurisdictional claims in published maps and institutional affiliations. 